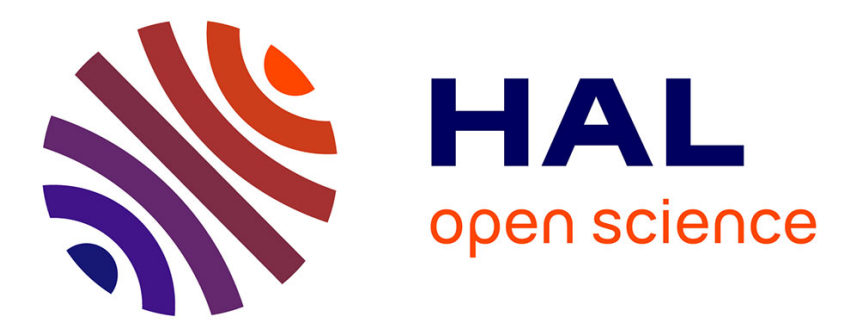

\title{
Non contact method for in vivo assessment of skin mechanical properties for assessing effect of ageing
}

Gaetan Boyer, Cyril Pailler-Mattéi, Jérôme Molimard, Marc Pericoi, Sabine Laquieze, Hassan Zahouani

\section{- To cite this version:}

Gaetan Boyer, Cyril Pailler-Mattéi, Jérôme Molimard, Marc Pericoi, Sabine Laquieze, et al.. Non contact method for in vivo assessment of skin mechanical properties for assessing effect of ageing. Medical Engineering \& Physics, 2012, 34 (2), pp.172-178. 10.1016/j.medengphy.2011.07.007 . hal00837958

\section{HAL Id: hal-00837958 \\ https://hal.science/hal-00837958}

Submitted on 14 Aug 2020

HAL is a multi-disciplinary open access archive for the deposit and dissemination of scientific research documents, whether they are published or not. The documents may come from teaching and research institutions in France or abroad, or from public or private research centers.
L'archive ouverte pluridisciplinaire HAL, est destinée au dépôt et à la diffusion de documents scientifiques de niveau recherche, publiés ou non, émanant des établissements d'enseignement et de recherche français ou étrangers, des laboratoires publics ou privés. 


\title{
Non contact method for in vivo assessment of skin mechanical properties for assessing effect of ageing
}

\author{
G. Boyer ${ }^{\mathrm{a}, *}$, C. Pailler Mattei ${ }^{\mathrm{b}, \mathrm{c}, * *}$, J. Molimard ${ }^{\mathrm{b}, \mathrm{d}}$, M. Pericoi $^{\mathrm{a}}$, S. Laquieze ${ }^{\mathrm{a}}$, H. Zahouani $^{\mathrm{b}, \mathrm{e}}$ \\ a Laboratoire Peritesco, 75001 Paris, France \\ b Université de Lyon, Laboratoire de Tribologie et Dynamique des Systèmes, UMR-CNRS 5513, Ecole Centrale de Lyon, 69130 Ecully, France \\ ' Université de Lyon, Université Lyon 1, ISPB, Laboratoire de biophysique, F-69003 Lyon, France \\ 'Centre SMS, Ecole des Mines de Saint-Etienne, 42023 Saint-Etienne, France \\ e Université de Lyon, Ecole Nationale des Ingénieurs de Saint-Etienne, 42023 Saint-Etienne, France
}

\begin{abstract}
The assessment of human tissue properties by objective and quantitative devices is very important to improve the understanding of its mechanical behaviour. The aim of this paper is to present a non contact method to measure the mechanical properties of human skin in vivo.

A complete non contact device using an air flow system has been developed. Validation and assessment of the method have been performed on inert visco-elastic material. An in vivo study on the forearm of two groups of healthy women aged of $23.2 \pm 1.6$ and $60.4 \pm 2.4$ has been performed. Main parameters assessed are presented and a first interpretation to evaluate the reduced Young's modulus is proposed.

Significant differences between the main parameters of the curve are shown with ageing. As tests were performed with different loads, the influence of the stress is also observed. We found a reduced Young's modulus with an air flow force of $10 \mathrm{mN}$ of $14.38 \pm 3.61 \mathrm{kPa}$ for the youngest group and $6.20 \pm 1.45 \mathrm{kPa}$ for the oldest group. These values agree with other studies using classical or dynamic indentation. Non contact test using the developed device gives convincing results.
\end{abstract}

\section{Introduction}

The knowledge of the human tissue mechanical properties is important for many research fields. Assessment performed by clinicians can be usefully completed by objective measurement by using specific devices which allow more reproducible results and make the comparison easier between studies performed by different teams. Information obtained can be used for example in the medical domain to compare healthy and diseased tissues, in order to continuously assess the evolution of a drug therapy. Another case where the objective measurement of the tissue properties is interesting is the cosmetic domain. The aim is here to compare the tissues before and after a product application in order to evaluate a potential improvement of the properties of the skin, generally rejuvenation. In this case, the knowledge of the properties of the skin with ageing is important in order to have a database for the

\footnotetext{
* Corresponding author at: PERITESCO, 7 rue Sérane, 34000 Montpellier, France. Tel.: +330467589860.

** Corresponding author at: Ecole Centrale de Lyon, Laboratoire de Tribologie et Dynamiques des Systèmes, 36 Avenue Guy de Collongue, 69134 Ecully, France. Tel.: +330472136291.

E-mail addresses: gaetan.boyer@peritesco.com (G. Boyer), cyril.pailler-mattei@ec-lyon.fr (C. Pailler Mattei).

URL: http://www.peritesco.com (G. Boyer)
}

comparison of the measurements with references. We see therefore that it is very important for the clinician to have accurate and reproducible devices. Many techniques exist to assess the mechanical properties of the human skin and its underlying tissues. We can mention indentation [1-3], suction [4,5], torsion [6-8], wave propagation [9-11] and extensometer [12-15] techniques. Non contact techniques based on air flow have been developed [16,17] more recently. The benefits of this technique are various. This method allows the assessment without any disturbance and preconditioning of the stressed area. Most of devices use double side adhesive tape to bond the probe to the skin. This adhesive tape induces modifications of the properties of the skin which forbids other measurement on the tested area, like moisture measurement or other mechanical assessments. Some sliding effects can also occur between the probe and the skin with the use of adhesive tape, which can induce measurement errors.

Moreover, the lack of plane contact area, due to a guard ring for example, allows measurement to any part of the body without any disturbances coming from shape modification. The aim of this paper is to propose a new specific non contact device based on a controlled air flow system and a high speed triangulation laser measurement. First, the device and the validation tests performed on inert material are presented. A semi-infinite linear elastic model allowing us to evaluate the apparent reduced Young's modulus is then proposed as a first approach. Second, the results obtained 


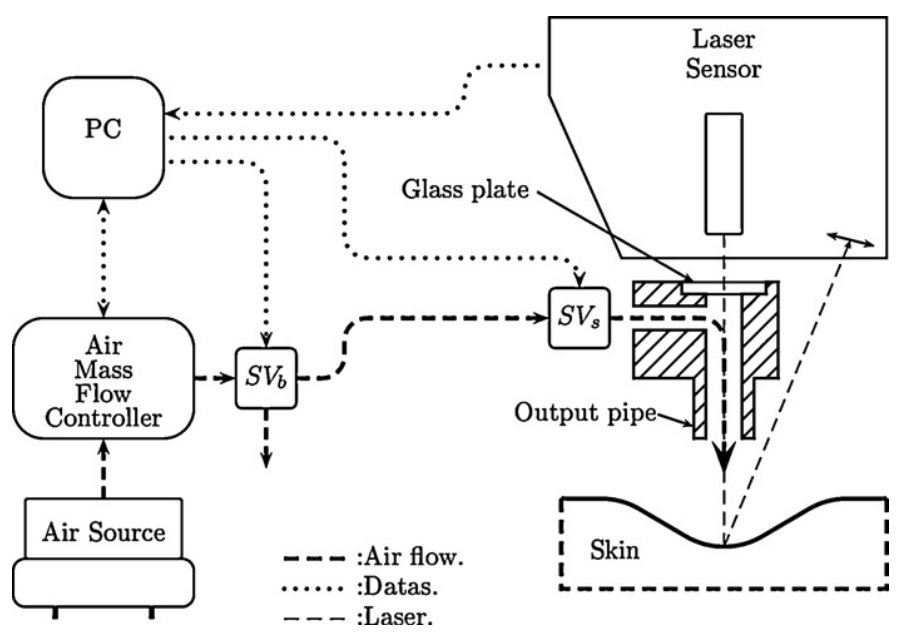

Fig. 1. Shema of the whole developed device.

during an in vivo study performed on 2 groups of young and old women were described. Evolution with ageing of measured parameters, comparison of the results with other studies and the perspectives of this work are finally discussed.

\section{Description of the experimental device}

The whole developed device called Tonoderm ${ }^{\circledR}$ (patent no. 08 05849, 22/10/2008) is shown in Fig. 1. The air flow is provided by an air compressor and is controlled by an Air Mass Flow Controller (AMFC) (5851S, Brooks Instrument) allowing a flow ranging from 2 to $100 \mathrm{l}_{\mathrm{n}} / \mathrm{min}$ (i.e. litre normalized per minute, the flow expressed in normalized pressure and temperature conditions). A first solenoid valve $S V_{b}$ placed at the output of the AMFC allows the bleeding of the air system. A second solenoid valve $S V_{S}$ is placed just before the output pipe and allows the instantaneous stop of the flow. It is important to put $S V_{S}$ as close as possible to the output in order to avoid a potential air tank in the pipe. The inner diameter of the output pipe is $2 \mathrm{~mm}$. The measurement of the gap between the end of the output pipe and the skin is performed using a Laser Displacement Sensor Head (LKG82, Keyence). The sample frequency of the sensor is $20 \mathrm{kHz}$ and its axial resolution is $1.5 \mu \mathrm{m}$. The diameter of the spot at a distance of $10 \mathrm{~mm}$ of the output pipe is about $70 \mu \mathrm{m}$. The laser beam passes through a glass plate allowing coaxiality between measurement and stress axis. Acquisition and generation of measurement and driving signals are performed using a 16-bit analog-digital I/O card (PCI-6221, National Instruments). A specific software developed under LabView ${ }^{\mathrm{TM}}$ language (National Instruments) allows to control the whole system.

The test performed is called "Ramp-Constant-Cut" (RCC) (Fig. 2). It consists on the application of a chosen force between the times $T_{0}$ and $T_{1}$. This force is then held during a time $T_{h}=T_{2}-T_{1}$ and is suddenly cut off using the solenoid valve $S V_{S}$ at $T_{2}$. The creeping of the material during the holding of the force and the free return after the instantaneous stop of the flow are observed. The following instantaneous parameters are measured: $Y d(\mathrm{~mm})$, the deflection after application of the force, $Y f(\mathrm{~mm})$, the deflection after the holding time, TG1 $(\mathrm{mm} / \mathrm{s})$, the instantaneous speed of the return of the skin, $T G 2(\mathrm{~mm} / \mathrm{s})$, the medium term speed of the return. The creeping $C_{r}(\mathrm{~mm})$ is also calculated $\left(C_{r}=Y f-Y d\right)$.

\section{Device validation}

The first step before using the device was to understand and to validate the stress applied by the air flow. For all tests and valida-

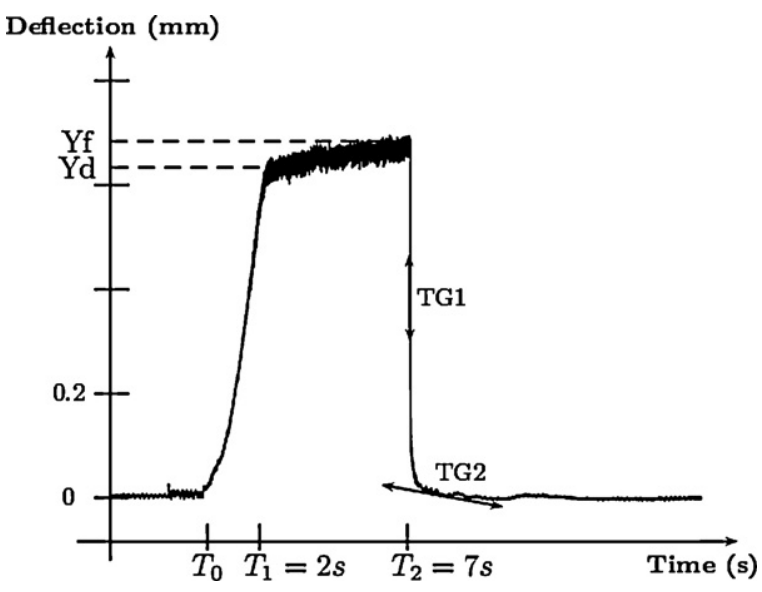

Fig. 2. Ramp-Constant-Cut test: typical curve obtained. $T_{0}$ is taken as $T=0$.

tions presented in this paper, the gap between the output pipe and the impact point of the air flow is $10 \mathrm{~mm}$.

\subsection{Pressure field and force applied by the air flow}

To characterize the air flow, the measurement of the pressure fields on a rigid plate has been performed using the assembly shown in Fig. 3.

A capillary tube (diameter: $380 \mu \mathrm{m}$ ) was fixed perpendicular to the plate, which is mounted on a micrometric translation stage. This translation stage allows tuning the distance $r$ between the axis of the air flow and the axis of the capillary tube. The tube is connected to a pressure sensor. Assessment of the pressure fields $p$ has been performed for a flow ranging from 6 to $20 \mathrm{l}_{\mathrm{n}} / \mathrm{min}$. Fig. 4 shows these fields.

They have been modelled by a function of the form:

$p=p_{0} a^{1-2 m}\left(a^{2}-r^{2}\right)^{m-(1 / 2)}$

with $p_{0}$ the pressure at the centre of the flow, $a$ the radius of the pressure area, $r$ the distance of a given point with the centre of the flow and $m$ an integer. We choose this form of pressure distribution according to the Boussinesq theory which gives the relationship between this kind of load and the Young's modulus. This relationship will be described in the following section. For more information on the pressure distribution see [18]. We

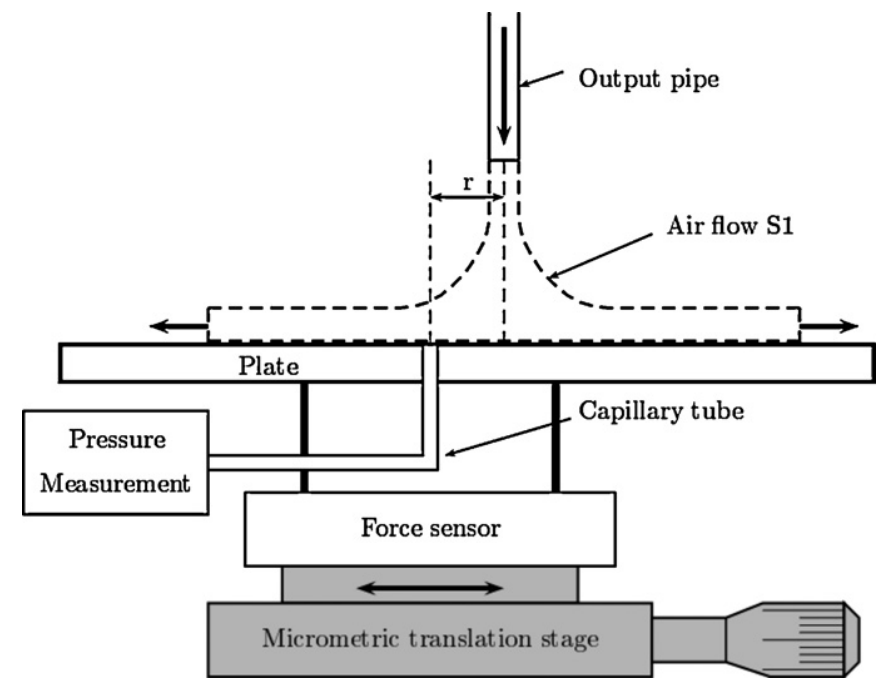

Fig. 3. Schema of the air flow impacting a plate. 


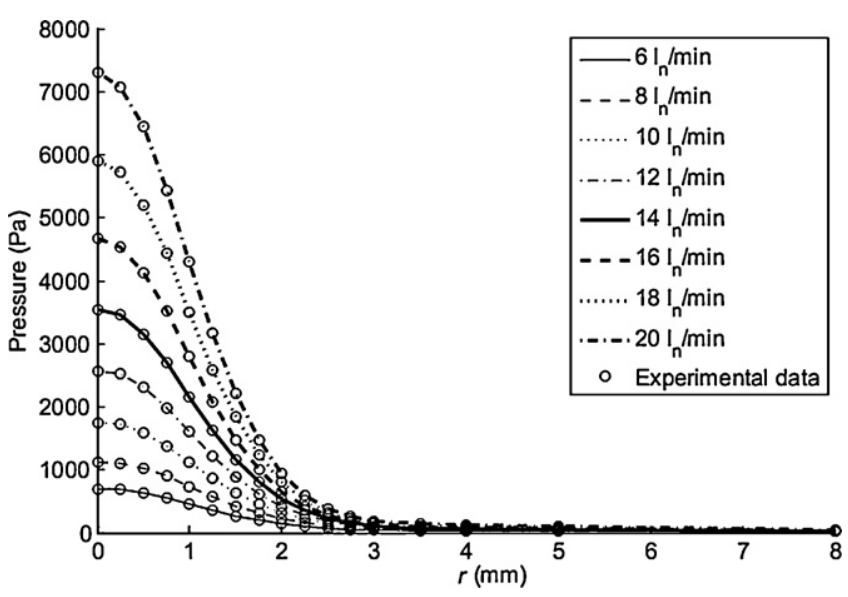

Fig. 4. Pressure fields applied on a plate.

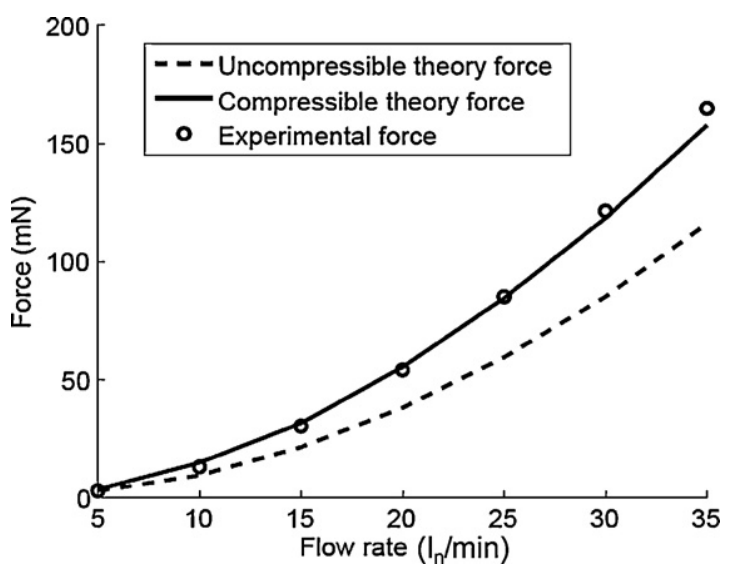

Fig. 5. Force as a function of flow rate. Comparison between theoretical force with uncompressible and compressible theory and experimental force.

consider a radius of the pressure area $a$ in Eq. (1) of $8 \mathrm{~mm}$, but values of pressure outside a $4 \mathrm{~mm}$ radius area is less than $5 \%$ of the maximal pressure.

To calculate the force applied by the air flow, the Euler theorem is used on the volume $S 1$ impacting a plate (Fig. 3). The total force applied is given by:

$F=Q_{m} v$

with $Q_{m}$ the mass flow and $v$ the flow velocity. In order to avoid experimental and fitting errors the experimental force is directly measured using a force sensor mounted on the rigid plate instead of using integration of Eq. (1). Due to the high velocity of the flow, the non compressible flow hypothesis cannot be applied. The velocity has also been corrected in Eq. (2) using the pressure measurement for $r=0$. This pressure has been related to the maximal velocity using thermodynamic equations, and the maximal velocity to the mean velocity using the form of the velocity field [19]. We have found good agreements between theoretical corrected force and experimental measurement, using a force sensor as shown in Fig. 5. This validation step permits the driving of the device in force unit instead of flow unit.

\subsection{Tests on inert material}

To validate the non contact device and to evaluate the Young's modulus of measured material, tests have been performed on a silicone plate with classical indentation test and non contact methods.

Assuming a semi infinite elastic material and a spherical indenter, the reduced Young's modulus, $E^{*}$, is given by [20,21]:

$E^{*}=\frac{K}{2 r_{c}}$

$E^{*}=\frac{E}{1-v^{2}}$

with $K$, the stiffness measured at the return of the indentation curve, $r_{c}$ the contact radius, $v$ the Poisson ratio and $E$ the Young's modulus of the indented material.

In the case of a non adhesive elastic material, $r_{c}$ is given by:

$r_{c}=\sqrt{\delta R}$

with $R$ the radius of curvature of the spherical indenter $(R=6.35 \mathrm{~mm})$ and $\delta$ the penetration depth.

In our case, the tested silicone is an adhesive material, that is the reason why the JKR theory [22] has been used to correctly estimate the contact radius, $r_{c, a d}$, between the indenter and the silicone:

$r_{c, a d}=\sqrt[3]{\frac{3 R}{4 E^{*}}\left(F_{N}+2 F_{a d}+2 \sqrt{F_{a d}\left(F_{N}+F_{a d}\right)}\right)}$

with $F_{N}$ the total applied force and $F_{a d}$ the adhesion force, which corresponds to the maximum measured negative force between the indenter and the material during the unloading part.

For the non contact device, the pressure field is given by Eq. (1). It can be shown that assuming this field, the displacement $u_{z}$ of a point at the surface of the material and at a distance $r$ of the centre of the flow is given by [18]:

$u_{z}=\frac{1-v^{2}}{\pi E^{*}} \frac{p_{0}}{a^{2 m-1}} \int_{0}^{2 \pi} \mathrm{d} \phi \int_{0}^{s 1}\left(\alpha^{2}-2 \beta s-s^{2}\right)^{m-(1 / 2)} \mathrm{d} s$

with

$\alpha^{2}=a^{2}-r^{2}$

$\beta=r \cos \phi$

$S_{1}$ is the positive root of $\alpha^{2}-2 \beta s-s^{2}$. In our case $r=0$ and $s_{1}=a$. Eq. (7) gives:

$u_{z}=\frac{\pi p_{0}}{E^{*} a^{2 m-1}} A_{1}$

$A_{1}=\int_{0}^{a}\left(a^{2}-s^{2}\right)^{m-(1 / 2)} \mathrm{d} s$

$A_{1}$ is numerically integrated. Coefficients $m$ and $p_{0}$ have been identified using a least squares method. Values obtained are given in Table 1.

Reduced Young's modulus measured on the silicone plate with forces ranging from 10 to $60 \mathrm{mN}$ is shown Fig. 6. Non adhesion and adhesion theories are shown for the indentation test. The measurement of Young's modulus using adhesion theory is closer to the non contact measurement than the non adhesion theory. The difference at $10 \mathrm{mN}$ force can be explained by the fact that the displacements

Table 1

Values of $m$ and $p_{0}$ obtained.

\begin{tabular}{|c|c|c|c|c|c|c|c|c|}
\hline Flow $\left(l_{n} / \min \right)$ & 6 & 8 & 10 & 12 & 14 & 16 & 18 & 20 \\
\hline$p_{0}$ & 670.98 & 1102.7 & 1732.0 & 2550.7 & 3513.7 & 4618.2 & 5848.3 & 7242.7 \\
\hline$m$ & 28 & 28 & 29 & 30 & 31 & 32 & 33 & 34 \\
\hline
\end{tabular}




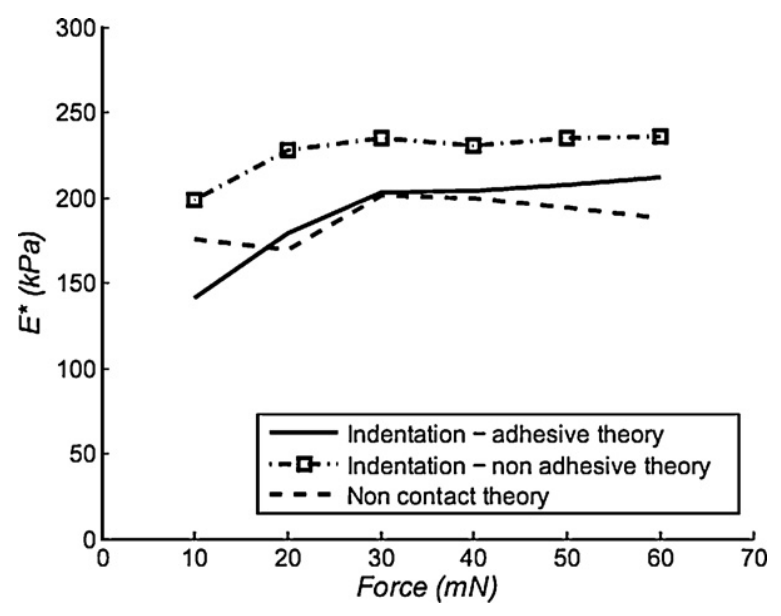

Fig. 6. Young's modulus measured on inert material. Comparison between Young's modulus calculated by indentation theory (non adhesive theory and adhesive theory) and Young's modulus calculated by non contact theory.

induced by the tests are very low compare to the device capabilities. A slight difference in the global trend for 50 and $60 \mathrm{mN}$ forces is observed. This difference can be explained because of the strains induced on the material for the high pressures probably modify the pressure field previously estimated on a rigid plate. Reduced Young's modulus found remains quite similar between both techniques and validates the non contact theory developed herein.

\section{Experimental procedure and results on human skin in vivo}

An in vivo study has been performed to evaluate the measurement using the non contact device. The procedure used is described below.

\subsection{Subject positioning}

The main drawback of the non contact technique is that the subject has to be static during the test. Because of the breath, heartbeats or unconscious movements, it is very difficult to measure low strain without immobilisation. A specific armrest has been developed allowing stiff fixation on the elbow and the wrist. The obtained disturbance movements are less than $30 \mu \mathrm{m}$ (Fig. 7).

\subsection{Subjects}

The measurements in vivo have been performed on 2 groups of healthy young and old women, respectively $23.2 \pm 1.6$ (group 1,14

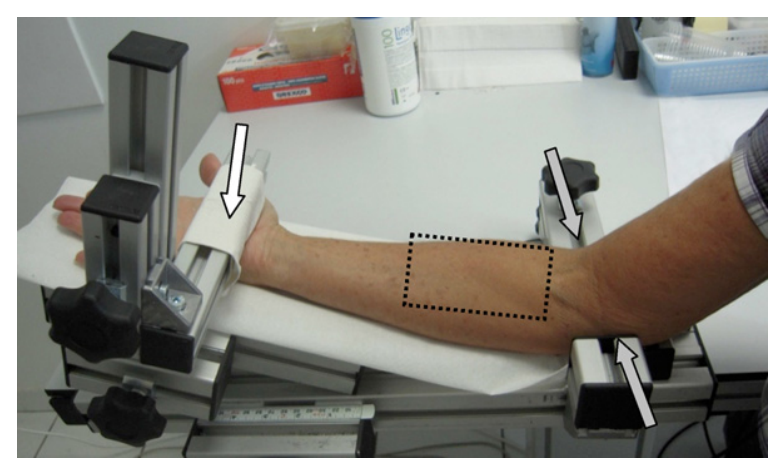

Fig. 7. Device used for test on the volar forearm. White arrow corresponds to the wrist fixation and grey arrows to the elbow fixation. Measurement area corresponds to the dashed frame. subjects) and $60.4 \pm 2.4$ (group 2, 14 subjects). All the volunteers have given their informed consent, they were free from pathological findings on their arms and had not use any topical agents on the tested area for $72 \mathrm{~h}$. The Body Mass Index was in the range of $20-25$, and no statistical difference between the two groups was observed.

\subsection{Protocol}

Measurements are made after 30 min of stabilization in a room with controlled temperature and humidity $\left(22^{\circ} \mathrm{C}\right.$ and RH $\left.50 \%\right)$. The subjects are seated in a medical chair with the specially designed armrest. The measurements are taken from the volar forearm at $60 \mathrm{~mm}$ from the elbow. RCC test was performed with time parameters: $T_{1}=2 \mathrm{~s}, T_{2}=7 \mathrm{~s}$ and recorded time after flow cutting of $10 \mathrm{~s}$. The forces applied were $10,20,30,40,50$ and $60 \mathrm{mN}$. The mean of 3 tests per each measurement has been done. After the non contact test, the thickness measurement was performed using a $25 \mathrm{MHz}$ echograph (Dermcup, Atys Medical).

\subsection{Statistical analysis}

Analysis of variance (ANOVA) has been performed to compare results between each group. A value of $P$ smaller than 0.05 is considered to be statistically significant and is represented with a star * over the data on each graphics.

\section{Results}

Fig. 8a and b show the evolution for each group of $Y f$ and $Y d$ with different forces. There is a statistically significant difference for each force except for $10 \mathrm{mN}$. Both parameters are higher for the old group, and we can see that there is an increase of the depth with the load. Fig. $8 \mathrm{c}$ shows the creeping $C_{r}$ for each group. We also observed a statistically significant difference for each force except $10 \mathrm{mN}$. We also observe an increase of the creeping with the load for both group and higher values for the old group. Fig. 8d and e show the evolution of TG1 and TG2 for each group with the force. TG2 has been calculated between a time of 0.1-0.2 s after the cut of the air flow. There is no statistically significant difference for TG1 except for $10 \mathrm{mN}$. There is an increase of this parameter with the load. We observed for TG2 an increase with the load and a statistically significant difference for each force except for $10 \mathrm{mN}$.

The Young's modulus for each group has been calculated using Eq. (10). We used $Y f$ value as $u_{z}$ in Eq. (1) in order to approach a quasi static test. Ideally, the time between $T_{1}$ and $T_{2}$ has to be sufficient for neglecting the viscosity. However due to the fact that we performed in vivo tests higher values of $T_{2}$ often induce uncontrolled movement and so unexploitable data. The evolution for each group with the force is shown on Fig. 8f. Statistical difference is observed except for $10 \mathrm{mN}$. This time an increase is observed for the young group, and a decrease is observed for the old group.

Note that measurements performed in vivo show that the coefficient of variation (standard deviation/mean $\times 100$ ) of the $Y f$ parameter on 5 tests is less than $1.5 \%$. The high variance/mean values observed in each group are mainly due to subject characteristics.

Table 2 shows the thickness of the dermis measured for each group. No statistical difference is observed.

Table 2

Skin thickness measured using the Dermcup echograph.

\begin{tabular}{ll}
\hline Group & Ep. $(\mathrm{mm})$ \\
\hline 1 & $1.094 \pm 0.128$ \\
2 & $1.033 \pm 0.108$ \\
\hline
\end{tabular}



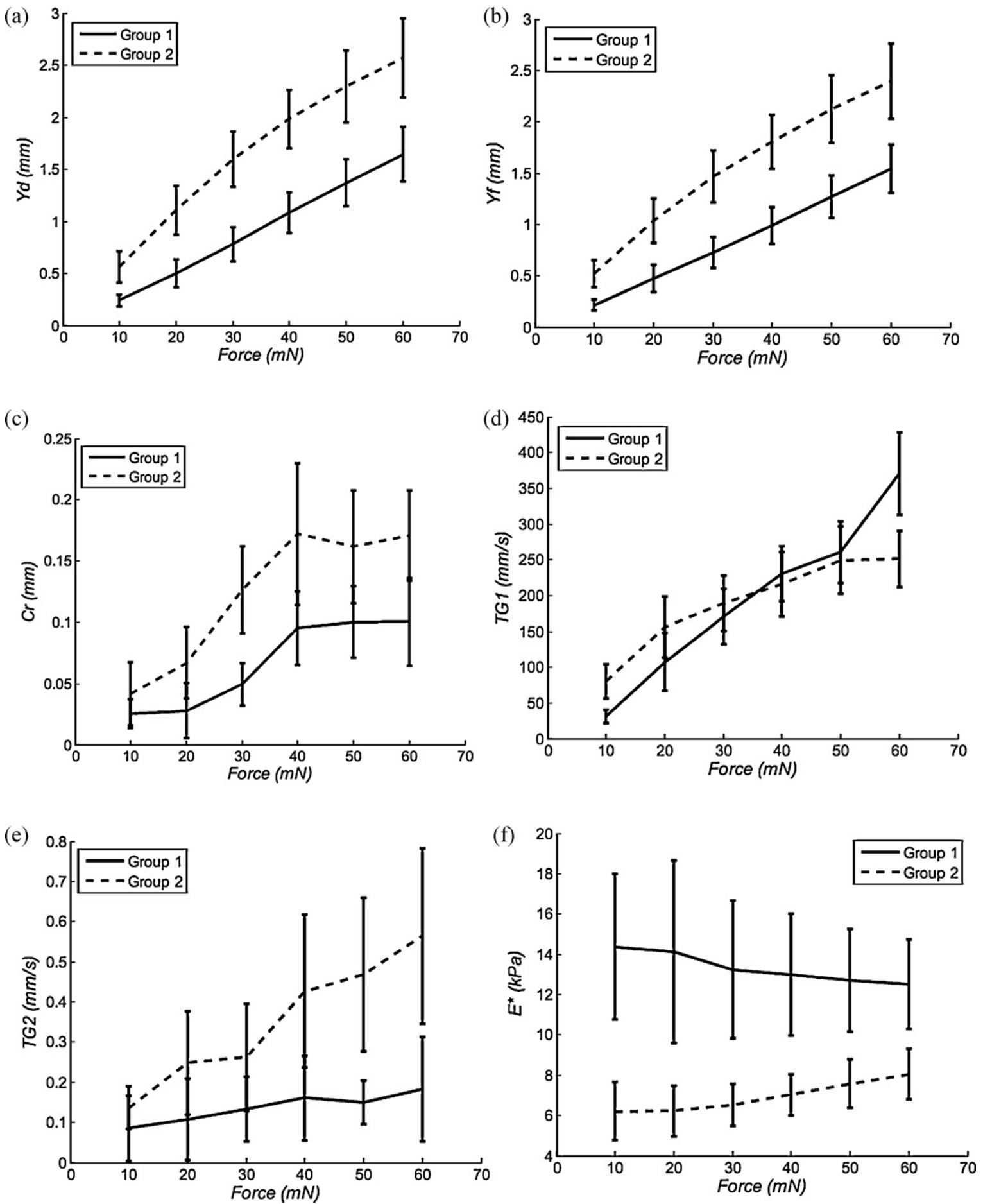

Fig. 8. Results of in vivo measurement on group 1 and group 2 .

\section{Discussion}

During this study, we observed that the quality of measurement using a non contact device is highly correlated to the quality of the subject immobilisation. The gap between the pipe and the skin has to be constant from the beginning to the end of the test. Our designed armrest reduces the disturbance movement to less than $30 \mu \mathrm{m}$. This disturbance movement can explain the lack of statistically significant difference at low strain for most parameters. We have a high ratio variance/mean, because disturbance movements represent $10 \%$ of the mean of $Y f$ and $Y d$ for group 1 . For measurement on other parts of the body, a lying down position will be probably the best. Problems will certainly occur in the case of measurement on the trunk due to the breath. In this case specific solutions will be searched.

$Y d$ parameter represents the elastic deflection of the skin. The value for group 2 is higher than the value for group 1 . It shows that the apparent stiffness of the contact is lower for the older group. It is interesting to notice that the evolution of $Y d$ with the load is more linear for the old group than the young group which traduces a difference of mechanical behaviour between young and old tissues with the depth. 
Yf parameter corresponds to the final value of the deflection. The same conclusion as $Y d$ can be done. The creeping after application of the load of the old group is more important than the creeping of the young group, but a similar evolution with the load is observed for both groups: the creeping remains constant from 40 to $60 \mathrm{mN}$ load. This behaviour is probably an effect of the subcutaneous tissues. TG1 is the short time recovery of the deflectance. The non significant difference for 30 and $40 \mathrm{mN}$ force is explained by the fact that the behaviour of the young and old tissues is different. For low load, the recovery speed is higher for the older group and for higher load, the recovery speed is higher for the younger group. This parameter represents the dynamic of the tissues. We demonstrate that the depth effect is important because we found different results at low and high load. TG2 is the long time recovery. The higher value for the old group show that the skin has not recovered all its strain and continues its recovering. The young skin shows more important elastic recovery and has also a lower long time recovery speed.

All these parameters show that the behaviour of the skin with ageing using non contact air flow device is very different. In order to take into account the shape of the load applied by the air flow, the reduced Young's modulus has been calculated. Due to the fact that no statistical difference on the skin thickness is observed and that the test is performed on the same area, we can say that the difference of behaviour is only due to the structure.

Validation and Young's modulus evaluation have been performed using a linear theory with the assumption of a plane semi-infinite body. The aim of this first approach is to evaluate the Young's modulus by taking into account the shape of the pressure field applied by the air flow. To evaluate the ageing effects a comparison of the measurements between the different groups have been performed. Because of the assumption of the flat surface of the material, the shape of the stressed area is not taken into account. It induces that the comparison between areas with different shapes cannot be performed. Most of the theories used by the different existing techniques make this assumption in a first way. As described below, we can see that the values of the Young's modulus obtained with the developed method give results in the same order as the other studies. This allows us to see that the assumptions performed are correct in the current study on the volar forearm. The model used needs to be improved and it should consider the shape of the skin that is not flat during the test and therefore disturbs the pressure field. The stress applied will change as a function of the maximum depth. The non linear behaviour and the thin multilayer structure of the material have also to be taken into account in order to have a realistic model. Another point that confirms the need of an improvement is that the area of pressure applied and the depth certainly induce a response of the subcutaneous layers, like the fat and muscle. Indeed, the higher the contact area is, the deeper the material is stressed. The effective contact area is equal to $4 \mathrm{~mm}$. More, the strain will be certainly different in the skin layer compare to the fat layer. The heterogeneity of the skin combined to the obtained depth show that the model used needs to be improved. Concerning the fat, we tried to minimize the influence of these subcutaneous tissues between the two groups by checking the Body Mass Index (no significant difference).

Another way to improve the current method is to evaluate the air flow pressure applied in a dynamic situation. The consideration of the evolution of the pressure field during the loading using an interaction fluid/structure numeric model for example should still improve the Young's modulus assessment. Due to the available material for the pressure measurement, the validation in this work has been only performed in a static state. This assessment will be certainly very complex due to the high velocity of the flow.

The main advantage of the non contact device compared to the classical contact device is to avoid to take into account the adhesion phenomena to estimate the contact area onto the stressed mate- rial. Other advantages coming from the lack of double side adhesive tape compared to suction or torsion test are also important to mention in the introduction. Tests can be performed on the same area several times without any disturbance, allowing more available measurements for the averaging of the measured parameters.

The in vivo results show that the modulus measured in a normal test is clearly higher for young people $(13-14.5 \mathrm{kPa})$ than for old people $(6-8 \mathrm{kPa})$. These results are in the same order of magnitude as literature values obtained by static indentation tests $[23,24]$, which are around $7-22 \mathrm{kPa}$ for women aged from 28 to 65 and obtained by dynamic indentation test [3], which are around $10.7 \pm 2.6 \mathrm{kPa}$ for the young people to $7.2 \pm 2.1 \mathrm{kPa}$ for the old people.

Concerning the evolution of the Young's modulus with the load, we can notice that there is no obvious trend, group 1 showing a slight decrease and group 2 a slight increase. This result disagrees with the results of Pailler-Mattei [25] with contact indentation test, showing a growth of the measured modulus with the load. A possible explanation could be the fact that the pressure fields used in Eq. (10) for the Young's modulus calculus have been assessed on a rigid plate. As mentioned above, the pressure fields are certainly influenced by the deformation of the surface of the measurement area. The method used in this study should be applied for small strain. The decrease of the Young's modulus with ageing agrees with results obtained by [3] showing that the non contact test is more sensitive to the natural tension of the skin: the skin becomes less tense with ageing. We can note that due to the fact that the viscosity is neglected, the Young's modulus is certainly overestimated.

In conclusion, this new non contact method gives convincing results for the assessment of the mechanical properties of the human skin in vivo. The non contact device avoids some problems as the adhesion effects that occur when using the contact tests or the disruption of the skin properties caused by the use of a double face adhesive that occurs in the suction or the torsion tests. The results obtained as a function of ageing and with the use of the developed model agree with the other studies. As said in the introduction, this kind of method can be very interesting for many research fields. However, the model should improve in the next studies in order to develop a more realistic model with the consideration of the multilayer structure, the non linear behaviour of the material and the evolution of the pressure field according to the shape of the tested area.

\section{Conflict of interest statement}

\section{No conflict of interest.}

\section{References}

[1] Pailler-Mattei C, Nicoli S, Pirot F, Vargiolu R, Zahouani H. A new approach to describe the skin surface physical properties in vivo. Colloids Surf B 2009;68:200-6.

[2] Pailler-Mattei C, Guerret-Piecourt C, Zahouani H, Nicoli S. Interpretation of the human skin biotribological behaviour after tape stripping. J R Soc Interface 2011;8:934-41.

[3] Boyer G, Laquièze L, Le Bot $A$, Laquièze $S$, Zahouani $H$. Dynamic indentation on human skin in vivo: ageing effects. Skin Res Technol 2009;15:55-67.

[4] Diridollou S, Vabrey V, Bersony M, Vaillantz L, Black D, Lagarde JM, et al. Skin ageing: changes of physical properties of human skin in vivo. Int J Cosmetic Sci 2001;23:353-62.

[5] Hendriks FM, Brokken D, van Eemeren J, Oomens CWJ, Baaijens FPT, Horsten JBAM. A numerical-experimental method to characterize the non linear mechanical behaviour of human skin. Skin Res Technol 2003;9:274-83.

[6] Sanders R. Torsional elasticity of human skin in vivo. Pflug Arch Eur J Phys 1973;342:255-60.

[7] Agache PG, Monneur C, Leveque JL, De Rigal J. Mechanical properties and Young's modulus of human skin in vivo. Arch Dermatol Res 1980;269:221-32.

[8] Berardesca E, de Rigal J, Leveque JL, Maibach HI. In vivo biophysical characterisation of skin physiological differences in races. Dermatologica 1991;182:89-93. 
[9] Moran CM, Bush NL, Bamber JC. Ultrasonic propagation properties of excised human skin. Ultrasound Med Biol 1995;21:1177-90.

[10] Pan L, Zan L, Foster FS. Ultrasonic and viscoelastic properties of skin under transverse mechanical stress in vitro. Ultrasound Med Biol 1998;24:995-1007.

[11] Gennisson J, Baldeweck T, Tanter M, Catheline S, Fink M, Sandrin L, et al. Assessment of elastic parameters of human skin using dynamic elastography. IEEE Trans Ultrason Ferroelectr Freq Control 2004;51:980-9.

[12] Alexander $\mathrm{H}$, Cook TH. Accounting for natural tension in the mechanical testing of human skin. J Invest Dermatol 1977;69:310-4.

[13] Ozyazgan I, Liman N, Dursun N, Güneş I. The effects of ovariectomy on the mechanical properties of skin in rats. Maturitas 2002;43:65-74.

[14] Del Prete Z, Antoniucci S, Hoffman A, Grigg P. Viscoelastic properties of skin in Mov-13 and Tsk mince. J Biomech 2004;37:1491-7.

[15] Lim K, Chew CM, Chen P, Jeyapalina S, Ho H, Rappel JK, Lim BH. New extensometer to measure in vivo uniaxial mechanical properties of human skin. J Biomech 2008;41:931-6.

[16] Fujimura T, Osanai O, Moriwaki S, Akazaki S, Takema Y. Development of a novel method to measure the elastic properties of skin including subcutaneous tissue: new age-related parameters and scope of application. Skin Res Technol 2008; 14:504-11.
[17] Fleury V, Al-Kilani A, Boryskina O, Cornelissen A, Nguyen TH, Unbekandt M, et al. Introducing the scanning air puff tonometer for biological studies. Phys Rev E 2010;81:021920.

[18] Johnson KL. Contact mechanics. England: Cambridge University Press; 2001

[19] Schlichting H, Gersten K. Boundary-layer theory. Berlin: Springer; 2000.

[20] Loubet JL, Georges JM, Marchesini O, Meille G. Vickers indentation curves of magnesium oxide. J Tribol 1984;106:43-8.

[21] Sneddon IN. The relation between load and penetration in the axiometric Boussinesq problem for a punch of arbitrary profile. Int J Eng Sci 1965;3: 47-57.

[22] Johnson KL, Kendall K, Roberts AD. Surface energy and the contact of elastic solids. Proc R Soc A: Math Phys 1971;324:301-13.

[23] Jachowicz J, McMullen R, Prettypaul D. Indentometric analysis of in vivo skin and comparison with artificial models. Skin Res Technol 2007;13: 299-309.

[24] Pailler-Mattei C, Zahouani H. Study of adhesion forces and mechanical properties of human skin in vivo. J Adhes Sci Technol 2004;18:1739-58.

[25] Pailler-Mattei C, Bec S, Zahouani H. In vivo measurements of the elastic mechanical properties of human skin by indentation tests. Med Eng Phys 2008;30:599-606 